Urologe 2018·57:269-271

https://doi.org/10.1007/s00120-018-0616-4

Online publiziert: 8. März 2018

(c) Springer Medizin Verlag $\mathrm{GmbH}$, ein Teil von Springer Nature 2018
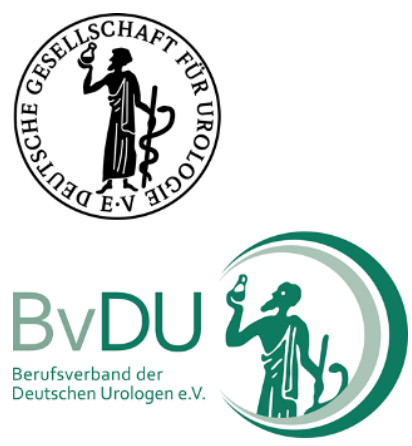

CrossMark

Auf Initiative und im Auftrag der Bundesärztekammer und der Privaten Krankenversicherung haben die Autoren ein Memorandum zur Diskussion einer Einheitlichen Gebührenordnung für Ärzte (EGO) verfasst, dessen Zusammenfassung wir Ihnen ungekürzt vorstellen.

Im Rahmen der Koalitionsverhandlungen zwischen CDU, CSU und SPD hat die SPD eine einheitliche Gebührenordnung auf die politische Agenda gesetzt. Eine einheitliche Gebührenordnung sei - so die Begründung - ein geeignetes Instrument, um u.a. oder wahlweise die "Zwei-Klassen-Medizin“, die „Zwei-Klassen-Wartezeiten“ oder die eingeschränkten Möglichkeiten der Inanspruchnahme medizinsicher Leistungen in strukturschwachen Regionen $\mathrm{zu}$ beseitigen respektive $\mathrm{zu}$ nivellieren. Das vorliegende Memorandum gibt einen Überblick darüber, warum die Vereinheitlichung der Vergütungsordnungen nicht nur ein in großen Teilen verfassungswidriges Unterfangen darstellt, sondern auch warum die mit

Der Generalsekretär der Deutschen Gesellschaft für Urologie, Prof. Dr. Maurice Stephan Michel, und der Präsident des Berufsverbandes der Deutschen Urologen, Dr. med. Axel Schroeder, haben vereinbart, besonders erwähnenswerte Nachrichten, die beide Verbände gleichermaßen betreffen, in einer gemeinsamen Kolumne an dieser exponierten Position der Verbandszeitschrift zu publizieren.

\author{
Rainer Hess ${ }^{1}$ Gregor Thüsing ${ }^{2}$. Volker Ulrich ${ }^{3}$ Eberhard Wille ${ }^{4}$. \\ Ferdinand Wollenschläger ${ }^{5}$ \\ 'Kanzlei für Medizin- und Gesundheitsrecht, Rechtsanwälte Hess und Partner PartGmbB, Köln, \\ Deutschland \\ ${ }^{2}$ Universität Bonn, Bonn, Deutschland \\ ${ }^{3}$ Universität Bayreuth, Bayreuth, Deutschland \\ ${ }^{4}$ Universität Mannheim, Mannheim, Deutschland \\ ${ }^{5}$ Universität Augsburg, Augsburg, Deutschland
}

\title{
Einheitliche Vergütung im dualen Krankenversicherungssystem?
}

einer einheitlichen Gebührenordnung verknüpften politischen Ziele nicht erreichbar sind und eine einheitliche $\mathrm{Ge}$ bührenordnung für die Versicherten bzw. für die Patienten mehr Nach- als Vorteile mit sich bringt. Die Ergebnisse im Überblick:

- Die historische Genese zeigt, dass sich Gebührenordnungen in Deutschland zwar stets im Korrektiv wechselseitig beeinflusst haben. Eine Konvergenz der Vergütungssystematik von GKV und PKV ist dabei aber nicht wahrzunehmen. Vielmehr folgt von Beginn an die Vergütungslogik der Systemlogik.

- Schon mit Blick auf die historische Entwicklung lässt sich die ärztliche Vergütung nicht nivellieren, ohne wesentliche Systemunterschiede zwischen GKV und PKV zu beseitigen. In anderen Worten: Die Systementscheidung geht der Vergütungslogik zeitlich, logisch und systematisch voran.

- Bei einer Wahl zwischen den beiden derzeitigen Gebührenordnungen dürfte der EBM im System der GKV mit rund 72,5 Mio. Versicherten gegenüber der GOÄ - zumindest in den Augen der Befürworter einer einheitlichen Gebührenordnung Priorität haben. Im Fall der GOÄ als einheitliche Gebührenordnung hingegen bestünde wegen deren Einzel- leistungsvergütung für die GKV die Gefahr einer erheblichen Mengenausweitung mit einem entsprechenden (zusätzlichen) Ausgabenwachstum.

- Eine einheitliche Gebührenordnung mit dem Regulierungsapparat des EBM (Budgetierung, Mengenbegrenzungen, Abstaffelungen etc.) wirft zahlreiche Fragen u.a. nach Kompensationszahlungen für die Leistungserbringer auf. Ohne Kompensation würde die Finanzierungbasis für medizinisches Personal und Investitionen fehlen. Die Versorgungslandschaft würde (weiter) ausgedünnt, die Versorgungqualität entsprechend sinken.

- Alternativ käme es - falls Honorarkürzungen politisch nicht mehrheitsfähig sind - zu einer Mehrbelastung der Beitragszahler. Die gesetzlich Krankenversicherten müssten dann die (ambulanten) Mehrumsätze der PKV-Versicherten tragen. Der Beitragssatz zur GKV würde sich um etwa 0,46 Prozentpunkte auf rund $16 \%$ erhöhen.

- Die Folge einer in einer einheitlichen Gebührenordnung inkludierten Mengensteuerung und Budgetierung nach Vorbild des EBM im GKV-System wäre ein sich schnell entwickelnder neuer Markt für „Premiumpatienten“. Denn niemand kann der PKV oder Ärzten - auch nicht 
der Gesetzgeber - verbieten, neue Zusatzangebote auf den Markt zu bringen, die z. B. einen Honorarzuschlag auf die einheitliche Vergütung nach sich ziehen würden. Teil dieses Zusatzangebots könnte explizit oder implizit u. a. auch eine kürzere Wartezeit sein. Diese Entwicklung hat sich auch in der Schweiz und in den Niederlanden ergeben, nachdem dort ein einheitlicher Versicherungsmarkt vom Gesetzgeber initiiert wurde.

- Das deutsche Gesundheitssystem weist im internationalen Vergleich durchgängig die geringsten Wartezeiten auf Arzttermine auf. Schon rein rechnerisch ist fragwürdig, was eine einheitliche Gebührenordnung verbessern könnte. Rund $10 \%$ Privatversicherte können die Wartezeiten der $90 \%$ gesetzlich Versicherten substantiell nicht verkürzen.

- Nicht das Nebeneinander von EBM und GOÄ, sondern die GKV-Budgetierung der ärztlichen Vergütung führt zu Wartezeiten - insbesondere am Ende des Quartals. Dieses „Quartalsphänomen“ wird von wissenschaftlichen Studien gestützt. Ende 2017 haben Gesundheitsökonomen auf Basis von Daten der Techniker Krankenkasse Zahlen vorlegen können, die zeigen, dass Ärzte immer am Ende eines Quartals weniger Patienten für einen Routinetermin annehmen. In der Konsequenz würden auch im Fall einer einheitlichen Gebührenordnung quartalsbedingte Wartezeiten entstehen, sofern diese - anlog zum EBM - weiter mit Mengensteuerung und Budgetierung operiert.

- Die derzeitigen Wartezeiten von GKV - und PKV-Versicherten bieten unter Versorgungsaspekten keinen relevanten Anlass für eine einheitliche Gebührenordnung. Auch bei Fortbestehen der beiden Gebührenordnungen gäbe es Möglichkeiten, die Wartezeiten der gesetzlich Versicherten zu verkürzen. So könnten z. B. selektive Verträge bestimmte Wartezeiten bindend vorsehen. Zudem könnte eine Änderung der quartalsbezogenen Pauschalen bestimmte fachärztlicher Leistungen die Wartezeiten verkürzen. Auch eine Reform der Bedarfsplanung könnte zur Verkürzung der Wartezeiten beitragen. Die Bedarfsplanung sollte zielgerichtet, bevölkerungsorientiert und sektorübergreifend ausgerichtet sein. Dabei ist das Morbiditätsniveau zu berücksichtigen.

- Die These, eine einheitliche Gebührenordnung würde Anreize schaffen, dass sich Ärzte stärker in strukturschwachen Regionen mit relativ wenig Privatpatienten niederlassen, überschätzt die Bedeutung der Honorare für die Standortwahl niedergelassener Ärzte. Für die Niederlassungsentscheidungen der ambulant tätigen Ärzte spielt, das zeigen Ergebnisse zahlreicher wissenschaftlicher Studien, das Umfeld für die Familie, Berufsalternativen für den Partner, Betreuungs- und Bildungsmöglichkeiten für Kinder, Verkehrsstruktur und Freizeitangebote eine viel entscheidendere Rolle.

- Der gute „Zustand“ des deutschen Gesundheitssystems schließt nicht aus, dass das Gesundheitssystem noch zahlreiche Möglichkeiten, insbesondere an den Schnittstellen der Leistungssektoren, für eine Verbesserung von Effizienz und Effektivität der Versorgung bietet. $\mathrm{Zu}$ den hierzu erforderlichen Reformen gehört eine einheitliche Gebührenordnung jedoch eindeutig nicht. Die Debatte um eine einheitliche Gebührenordnung lenkt eher von den eigentlichen Problemen und Verbesserungsmöglichkeiten des deutschen Gesundheitswesens ab.

- Die Vereinheitlichung der kassenund privatärztlichen Vergütungen wäre zudem aus rechtlicher Sicht ein verfassungswidriges und gegebenenfalls auch ein europarechtswidriges Unterfangen. In die Vertragsfreiheit der Versicherten und in die Berufsfreiheit der Ärzte sowie der Krankenversicherer würde eingegriffen werden, ohne dass ausreichende Rechtfertigungsgründe ersichtlich wären, die den Maßstäben des Bundesverfassungsgerichts genügen würden.
- Mit der Vereinheitlichung der kassenund privatärztlichen Vergütungen würde ein Mischsystem geschaffen, das auch unter Gleichheitsgesichtspunkten ebenso wie unter dem Aspekt der Systemstimmigkeit und der Vereinbarkeit mit den vorhandenen Institutionen der gemeinsamen Selbstverwaltung erhebliche Fragen aufwürfe.

\section{Fazit}

Die Forderung nach einer einheitlichen Gebührenordnung ohne Mengensteuerung und Budgetierung nach dem Vorbild der Einzelvergütung in der Gebührenordnung für Ärzte birgt für den Gesetz- und Verordnungsgeber das Risiko von Kosten- und Beitragssteigerungen. Auch mit Blick auf die 40\%-Grenze, die die Sozialversicherungsbeiträge in der Summe nicht übersteigen sollte, schüfe eine derartige „Vereinheitlichungsrichtung" eher Probleme als Probleme zu lösen. Mit Blick auf die Finanzierbarkeit der GKV dürfte eine einheitliche Gebührenordnung sich vielmehr an den Mengen- und Preisregulierungen des GKV-Systems orientieren. Dies würde der medizinischen Infrastruktur nicht nur erhebliche finanzielle Mittel entziehen, sondern auch einen ordnungspolitischen wie rechtlich fragwürdigen Systembruch bedeuten. Zugleich würde man dem „Ideal“ der Gleichheit nicht näher kommen: In der Folge würde sich schnell ein neuer Zusatzversicherungsmarkt etablieren, der all diejenigen Leistungs- und Preisdifferenzierungen erneut wiedereinführt, die vom Gesetz- und Verordnungsgeber durch die einheitliche Gebührenordnung gerade vermieden werden sollten.

\section{Korrespondenzadresse}

\section{Dr. R. Hess}

Kanzlei für Medizin- und Gesundheitsrecht, Rechtsanwälte Hess und Partner PartGmbB Ehrenstr. 45-47, 50672 Köln, Deutschland r.hess@hess-anwaelte.de

Interessenkonflikt. R. Hess, G. Thüsing, V. Ulrich E. Wille und F. Wollenschläger geben an, dass kein Interessenkonflikt besteht. 
Hier steht eine Anzeige.

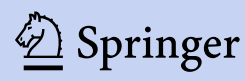

